\title{
EDITORIAL
}

\section{NURSING HOMES AND LONG TERM CARE AFTER COVID-19: A NEW ERA?}

\author{
M. INZITARI ${ }^{1,2,3}$, E. RISCO ${ }^{1}$, M. CESARI ${ }^{4}$, B.M. BUURMAN ${ }^{5}$, K. KULUSKI ${ }^{6}$, V. DAVEY ${ }^{1}$, L. BENNETT ${ }^{7}$, \\ J. VARELA ${ }^{8}$, J. PRVU BETTGER ${ }^{9}$
}

\begin{abstract}
1. REFiT Barcelona research group, Parc Sanitari Pere Virgili and Vall d'Hebron Institute of Research (VHIR), Barcelona, Spain; 2. Universitat Autònoma de Barcelona, Barcelona, Spain; 3. Fundació Tecnocampus, Mataró, Barcelona, Spain; 4. Policlinico Maggiore Hospital and Università Statale, Milan, Italy; 5. Amsterdam UMC, University of Amsterdam,

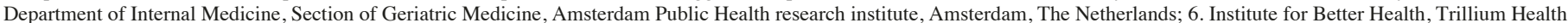

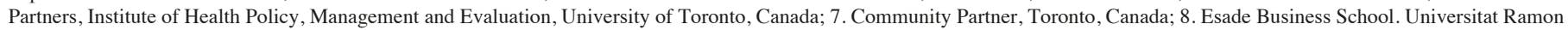

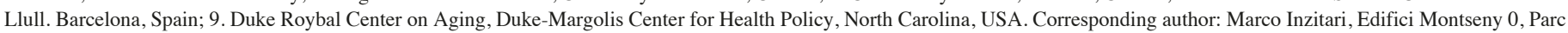
Sanitari Pere Virgili, c Esteve Terradas 30,08023 Barcelona, Spain, Email: minzitari@ perevirgili.cat, Phone: +34 932594004
\end{abstract}

\section{Main text}

Anna, 92 years old, former housekeeper, with a history of disabling stroke and disabling knee osteoarthritis, had been living in a Nursing Home $(\mathrm{NH})$ for the last 6 years. In Anna's NH, $60 \%$ of the staff were affected by COVID-19. In March 2020, she was transferred to hospital due to hyperactive delirium where she was diagnosed with COVID-19 but then transferred to an alternative $\mathrm{NH}$, designated for COVID-19 care. She died 2 days later having received palliative care but in unfamiliar surroundings. From the time her original $\mathrm{NH}$ was closed to visitors, until her death, she did not see any family members.

Amanda, 87 years old, a widow and former schoolteacher, was diagnosed with Alzheimer's disease 8 years ago and moved to a nursing home 2 years ago. Prior to the outbreak she was able to follow a basic conversation and had limited mobility with a walker, participated in some group activities and had regular family visitors. She died after one month isolated in her room, initially because she had been in contact with another resident diagnosed with COVID-19 and later because of her deterioration. She did not experience the hallmark symptoms of COVID-19, and had a negative PCR for SARS-COV-2, but she became progressively more disoriented and spent more time in bed.

\section{Threats and preparation for new outbreaks}

These two stories could have happened in Spain, Italy, the Netherlands, the USA or Canada. They reflect some of the potential health and psychological consequences, directly or indirectly brought on by COVID-19, to older adults living in long-term care (LTC) facilities, including nursing and residential homes, hereafter referred to as nursing homes $(\mathrm{NH})$. NH residents have been the most affected by COVID19 in many countries (1), representing as many as half of all deaths for COVID-19 in a number of European countries, over three quarters in Canada and around $40 \%$ in the USA, according to some of the latest available data sources (Figure $1)$. Despite cross and within-country heterogeneity in policies, responsibilities and funding for long-term care (2), NHs share many common threads in infrastructure, organization and workforce (3), including low staff to resident ratios; low paid staff; low skill-mix and high staff turnover, creating environments with minimal resilience to adverse events.

Critical errors have been replicated by different countries, despite initial warnings (5), including the lack of prioritization of NHs for the organization and provision of protective measures (PPEs), delays in testing of workers and patients and support staff shortages $(3,4)$, contributing to the spread of infection and the capacity to meet infection control protocols and provide adequate care. In most countries, deaths have mainly occurred within the NHs themselves, as, during the most critical phases of the pandemic, admission criteria have limited access of NH residents to secondary care. This may have contributed to further spreading SARS-CoV-2 within facilities and has led to questions about levels of medical and palliative care, with palliative care and access to integrated medical care already undeveloped (6). There are very few international examples of actions to increase health care, either within homes $(7,8)$ or by providing care in hospitals or sub-acute facilities (9) during the crisis.

COVID-19 has shown us that during a pandemic LTC facilities such as NHs require adequate PPEs (and training in its use), quick diagnostic testing and measures to alleviate staff shortages, which account for additional demands and include the provision of reinforcement teams (8). Protocols for infection control rely on sufficient space to allow social distancing (10) and isolation, but many countries still rely heavily on physical structures where shared rooms are common. Nevertheless, isolation needs to be balanced with the need to avoid immobility and maintain social and family connections (where technology can provide some solutions but may not be suitable for residents with advanced dementia) (11).

The growing COVID-19 death toll in NHs internationally has resurfaced the discussion on care, quality, workforce and living conditions. It has also undermined public confidence in $\mathrm{NHs}$, which were experiencing problems long before this crisis. Compared with same-aged community dwelling older adults, nursing home residents accumulate more clinical complexity, multi-morbidity and frailty overtime (12). The USA now has lower nursing home occupancy because alternative models Published online July 11, 2020, http://10.1007/s12603-020-1447-8 
Figure 1

Estimations of deaths in LTC facilities out of total deaths caused by COVID-19

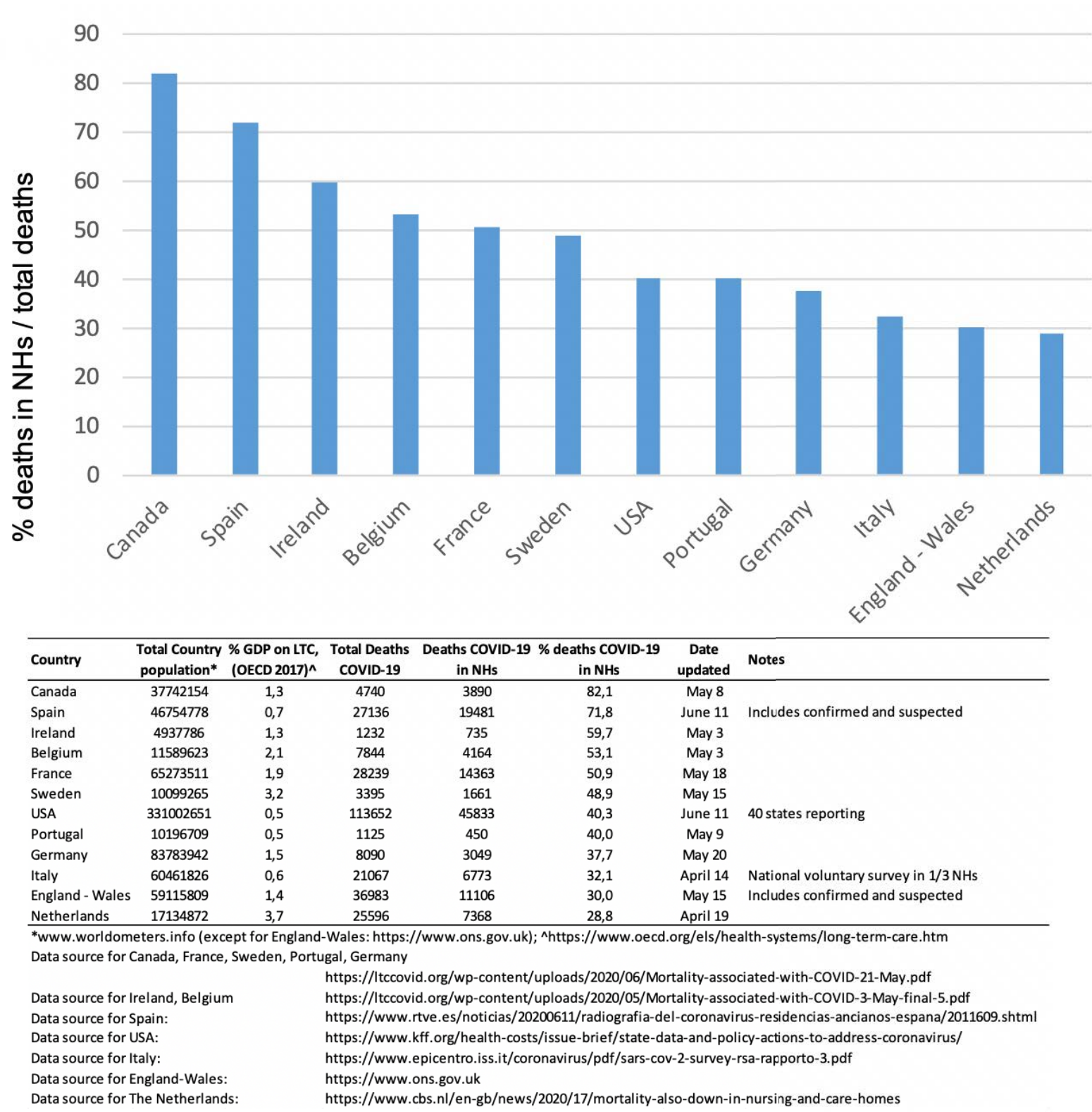

for long-term services and supports are often better suited to meet older adults' preferences and needs (13). In other countries though, underdeveloped home care and lack of integrated support for people with complex care needs, has led to increased demand, overwhelming LTC facilities and subsequently leading to more hospitalizations (2). These challenges highlight the need for a deep, open, societal discussion on the future of LTC facilities, and long-term care models for older adults. This pandemic might represent, at least, an opportunity to redesign a sustainable model (4).

\section{Home as the place to stay}

Multiple surveys suggest that most older adults prefer to remain in their homes as long as possible (14). For people who decide or need to move for health or socio-economic needs, co-housing, sheltered housing or assisted living are valuable alternatives, although these options are not always viable with transitions across the boundary between the third and fourth age and adaptation to increasing care needs proving challenging (15).

In the US, the Program of All-Inclusive Care for the Elderly (PACE) has been effective in supporting older adults at home 
who would otherwise fulfill the criteria to be institutionalized (16), through integrated provision of health and social care after a comprehensive assessment of needs, at a single entry point. The community nursing care model in the Netherlands (epitomized by Buurtzorg) enables older people to remain at home by provision of community nursing services, which can be highly intensive (17) and facilitates specialist geriatric care at home in case of complex problems or crises. To achieve this, investments in community nursing and specialist intermediate care services at home (18) are essential.

During the pandemic, we have learned that $\mathrm{NHs}$, in their current form and with current resources and priority applied to them (for PPE, testing, medical support), are an unsafe place to remain. In addition, collective decisions, such as the restriction of visits, have had a major impact on residents and caregivers' quality of life, causing much grief, especially at the end of life. While home-based options may permit better containment of transmission $(19,20)$, to achieve this community-based care workers also require adequate protection (PPE), job security and wages. Rethinking $\mathrm{NH}$ care might mean that home is the safest place, but also the place where older persons can decide for themselves what risks are acceptable and quality of life can be achieved. Although a strategy towards deinstitutionalization of care is not new, in many countries, within the EU, it has been under-developed, while the number of long-term care beds has increased (2).

\section{Improving quality and person-centeredness in NHs is urgent}

If home-care is the base of the future system, is there a role for institutional-based care? The answer relies, in part, on the ability to provide effective, accessible and affordable home and community-based care and support unpaid carers. Also, the speed and depth of change will likely be context-dependent (2), and related to social and housing policies. In the case of people with very complex needs, such as people with latestage dementia, home care might not always be a viable option. Depending on the situation, moving to a $\mathrm{NH}$ is not necessarily associated with a decreased quality of life (21). Certainly, $\mathrm{NHs}$ need new policies, models and investments including:

- person and relationship-centered care allowing real flexibility and choices to improve quality of care (22), shared decision-making, meaningful activities and social interactions (23), adaptation to the needs of people with dementia (24), promotion of healthier lifestyles, and embracement of new technologies which hold the potential to increase quality of life in $\mathrm{NHs}$;

- participation of older people and their caregivers in the concept, design, implementation and oversight of NHs (25);

- an "age-friendly" environment, with enough space to guarantee privacy (and potentially to facilitate segmentation during a pandemic) and well-designed outdoor spaces;

- an adequate size: smaller or middle-scale homelike structures would avoid the excessive standardization of routines of large-scale institutions (26);

- training and support for nursing aides or assistants (also named auxiliary nurses or personal support workers), recreational therapists and volunteers, who also play a key role;

- robust, continued healthcare, with 24 hour registered nurses, plus central roles for physical and occupational-therapists, social workers, psychologists, nutritionists and pharmacists. Any medical support (even with ad-hoc trained figures such as in the Dutch model) needs to be integrated with community-based services and support;

individualized care plans, focused on secondary and tertiary prevention and rehabilitation and early palliative care;

- clear care pathways linking NHs to intermediate or postacute care, (where available) and to acute care, to prevent residents from being overlooked or discriminated upon in access to health care of the basis of age, or place of residence;

- standardized tools for comprehensive, bio-psycho-social assessment of the resident (including functional capacity and frailty) and agreed outcomes, in order to monitor health trajectories and provide data of public health interest.

\section{Principles to reshape long term care}

Independent of the setting (private home or nursing home), there are shared principles to take into account. Different evidence-based and policy documents support the main pillars of a new model for long-term care, summarized in Table 1 . Older persons' preferences and the active involvement of them and their caregivers', must be the starting point (27). Integrated health and social care should be modelled accordingly, with prevention of disability and rehabilitation as common aims (2). Continuity of care on a daily-basis by trained professionals should be integrated with primary care resources. Specialists in geriatrics and gerontology (16) should be involved in both conceptual and management aspects, as well as providing care in cases of complex clinical needs and for specialized timelimited interventions at home during health crises (18). At an individual level, each older person deserves an individualized care plan and an advanced care plan, based on a comprehensive geriatric assessment, using principles of person-centered care and through a shared decision making approach (28). At a structural level, investments for professional training to improve quality of care, as well as defining and monitoring care standards are crucial (2). Telemedicine solutions add value for continuing education, consultation, support and benchmarking (29).

No doubt, pragmatic economic and financial considerations will shape model orientation and implementation for each context. The risk is that decisions will be driven on absolute costs within a narrow paradigm, such as reduction of hospital care or readmissions, rather than value based analyses or 
THE JOURNAL OF NUTRITION, HEALTH \& AGING

Table 1

Pillars to redefine long term care post COVID-19

\section{Older people and caregivers' involvement}

- The new model should be rooted in older peoples' preferences

- Older persons and their caregivers should participate in the concept, design, implementation and oversight of the model.

\section{Delivery of care}

- Care at home should be accessible and affordable, and alternatives to home, including care and nursing homes, should be homelike.

- Adequate health and social care should be integrated.

- Continuity of care with primary health and social care is warranted.

- Specialists in geriatrics and gerontology should be involved in the design, management and overview.

- Geriatricians should provide additional support for complex clinical needs (e.g. severe dementia) or time-limited interventions during health crises.

\section{Care at an individual level}

- Patient-centered care is pivotal across all aspects (personal care, activities, spaces should reflect person's preferences).

- Each person deserves an individualized care plan and an advanced care plan, based on a comprehensive geriatric assessment and shared decision-making.

- Prevention and rehabilitation, as well as early palliative care, are key.

\section{Organizational aspects}

- Investments in professional training and standardized monitoring of the extent to which care meets complex needs are relevant to improve quality.

- Frameworks for auditing person and relationship-centered care should be coproduced by people who receive long-term care.

- Telemedicine solutions are a valuable wide-scale support for continuing education, consultation, support and benchmarking.

decisions (cost-quality or whole system impact). Also, if these solutions are not planned at a system level and provided at an acceptable cost, disparities, based on the socio-economic status of older people, will increase. Finally, other variables, such as the role of informal care, which is highly context and culturespecific, will influence the final model.

\section{Conclusions}

COVID-19 provides an opportunity to address chronically overlooked and underfunded sectors of long-term care, including nursing and residential homes, and homecare. Each mode of delivering long-term care must provide optimal standards of continuing care and maximum quality of life. More evaluation and research is needed to support decision and policy-making, in particular on the cost-effectiveness and cost-quality aspects for each specific country, region or system. So far, evidence on what works seems to point to person and relationship-centered solutions, combined with integrated health and social care, plus telehealth, to ensure timely, individualized responses to persons' needs.

Contributors and sources: This work is the result of an international collaboration, between experts on geriatrics (Inzitari, Risco, Cesari, Buurman), long term care policy (Davey, Prvu Bettger), gerontological research (Kuluski, Prvu Bettger), healthcare management applied to older adults with complex needs (Varela, Inzitari) and caregivers of persons with dementia (Bennett), based in EU countries, in the UK and in North America. All of these countries are experiencing a disproportionate rate of infection and therefore greater impact of COVID-19 in their care homes. All the co-authors actively participated in designing and writing the manuscript, and in adding relevant evidence; Lisa Bennett has reviewed and improved the manuscript from the caregivers' perspective; Inzitari, Davey and Prvu Bettger collected the data on mortality in care homes. Marco Inzitari acts as guarantor of the article.
Patient involvement: This manuscript is co-authored by Lisa Bennett, a caregiver of a person with dementia living in a care home, from in Toronto, Canada.

Conflicts of Interest: We have read and understood Journal of Nutrition, Health \& Ageing policy on declaration of interests and have no interests to declare related to this manuscript.

\section{References}

1. Comas-Herrera A, Zalakaín J, Litwin C, Hsu A, Lane N, Fernández J. Mortality associated with COVID19 outbreaks in care homes: early international evidence. 2020. https://ltccovid.org/wp-content/uploads/2020/05/Mortality-associated-with-COVID-3 May-final-2.pdf. Accessed 7th June 2020.

2. Spasova, S., Baeten, R., Coster, S., Ghailani, D., Peña-Casas, R. and Vanhercke B. 2018 Challenges in long-term care in Europe. A study of national policies. https:// www.age-platform.eu/publications/challenges-long-term-care-europe-study-nationalpolicies-2018. Accessed 28th May 2020.

3. McMichael TM, Currie DW, Clark S, Pogosjans S, Kay M, Schwartz NG, et al. Epidemiology of Covid-19 in a Long-Term Care Facility in King County, Washington. N Engl J Med 2020; 382:2005-2011.

4. Barnett ML, Grabowski DC. (2020) Nursing Homes Are Ground Zero for COVID-19 Pandemic. JAMA Healh Forum 2020 [epub]:e200369-e200369.

5. WHO/Europe I Media centre. Invest in the overlooked and unsung: build sustainable people-centred long-term care in the wake of COVID-19. 2020. http://www.euro.who. int/en/media-centre/sections/statements/2020/statement-invest-in-the-overlooked-andunsung-build-sustainable-people-centred-long-term-care-in-the-wake-of-covid-19. Accessed 18th May 2020.

6. Joni G, Lara P, Unroe Kathleen T, Lieve V den B. International COVID-19 palliative care guidance for nursing homes leaves key themes unaddressed. J Pain Symptom Manage. 2020; doi:10.1016/j.jpainsymman.2020.04.151

7. Rolland Y, Benetos A, Villars H, Braun H, Blain H. A COVID-19 Support Platform for Long Term Care Facilities. Vol. 24, J Nutr Health Ageing2020;24:461-462. doi 10.1007/s12603-020-1364-x

8. Stall NM, Farquharson C, Fan-Lun C, Wiesenfeld L, Loftus CA, Kain D, et al. A Hospital Partnership with a Nursing Home Experiencing a COVID-19 Outbreak: Description of a Multiphase Emergency Response in Toronto, Canada. J Am Geriatr Soc Jun 13 2020; doi:10.1111/jgs.16625.

9. Inzitari M, Udina C, Len O, Ars J, Arnal C, Badani H, et al. How a Barcelona postacute facility became a referral center for comprehensive management of subacute patients with COVID-19. J Am Med Dir Assoc Med Dir Assoc, Jun 172020 


\section{NURSING HOMES AND LONG TERM CARE AFTER COVID-19: A NEW ERA?}

doi:10.1016/j.jamda.2020.06.015

10. Abrams HR, Loomer L, Gandhi A, Grabowski DC. Characteristics of U.S. Nursing Homes with COVID-19 Cases. J Am Geriatr Soc. Jun 4 202. doi:10.1111/jgs.16661

11. Simard J. Loneliness and Isolation in Long-term Care and the COVID-19 Pandemic. J Am Med Dir Assoc, June 17 2020, doi:10.1016/j.jamda.2020.05.006

12. Ng R, Lane N, Tanuseputro P, Mojaverian N, Talarico R, Wodchis WP, et al Increasing Complexity of New Nursing Home Residents in Ontario, Canada: A Serial Cross-Sectional Study. J Am Geriatr Soc 2020;; 68:1293-1300.

13. The New York Times. In the Nursing Home, Empty Beds and Quiet Halls. 2020 https://www.nytimes.com/2018/09/28/health/nursing-homes-occupancy.html. Accessed 15th May 20220.

14. AARP. Survey: 75 Percent of Retirees Want to Stay Put. 2020. https://www.aarp.org/ retirement/planning-for-retirement/info-2018/retirees-age-in-place-aarp-study.html. Accessed 15th May 2020

15. Johnson EK, Cameron A, Lloyd L, Evans S, Darton R, Smith R, et al. Ageing in extra-care housing: Preparation, persistence and self-management at the boundary between the third and fourth age. Ageing Soc. Jul 18 2019, doi:10.1017/ S0144686X190008491-21.

16. Mukamel DB, Peterson DR, Temkin-Greener H, Delavan R, Gross D, Kunitz SJ, et al. Program Characteristics and Enrollees' Outcomes in the Program of All-Inclusive Care for the Elderly (PACE). Milbank Q 2007; 85:499-531.

17. Drennan VM, Calestani M, Ross F, Saunders M, West P. Tackling the workforce crisis in district nursing: Can the Dutch Buurtzorg model offer a solution and a better patient experience? A mixed methods case study. BMJ Open 2018; 8:6.

18. Mas MÀ, Inzitari M, Sabaté S, Santaeugènia SJ, Miralles R. Hospital-at-home Integrated Care Programme for the management of disabling health crises in older patients: Comparison with bed-based Intermediate Care. Age Ageing 2017; 46:92531.

19. Nacoti M, Ciocca A, Giupponi A, Brambillasca P, Lussana F, Pisano M, et al. At the Epicenter of the Covid-19 Pandemic and Humanitarian Crises in Italy: Changing Perspectives on Preparation and Mitigation. New Eng Jnl Med Catalyst 2020 doi:10.1056/CAT.20.0080
20. Roxby AC, Greninger AL, Hatfield KM, Lynch JB, Dellit TH, James A, et al Detection of SARS-CoV-2 Among Residents and Staff Members of an Independent and Assisted Living Community for Older Adults - Seattle, Washington, 2020 MMWR Morb Mortal Wkly Rep 2020; 69:416-8.

21. Beerens HC, Zwakhalen SMG, Verbeek H, Ruwaard D, Ambergen AW, Leino-Kilp $\mathrm{H}$, et al. Change in quality of life of people with dementia recently admitted to long term care facilities. J Adv Nurs 2015; 71:1435-47.

22. Grabowski DC, James O'malley A, Afendulis CC, Caudry DJ, Elliot A, Zimmerman S. Culture Change and Nursing Home Quality of Care. Gerontologist 2014; 54:35-45.

23. Adlbrecht L, Bartholomeyczik S, Hildebrandt C, Mayer H. Social interactions of persons with dementia living in special care units in long-term care: A mixed-methods systematic review. Dementia. Apr 23 2020, doi: 10.1177/1471301220919937.

24. Galik E, Resnick B, Hammersla M, Brightwater J. Optimizing Function and Physical Activity Among Nursing Home Residents With Dementia: Testing the Impact of Function-Focused Care. Gerontologist 2014; 54:930-43.

25. de Graaff MB, Stoopendaal A, Leistikow I. Transforming clients into experts-byexperience: A pilot in client participation in Dutch long-term elderly care homes inspectorate supervision. Health Pol 2019; 123:275-80.

26. Ausserhofer D, Deschodt M, De Geest S, van Achterberg T, Meyer G, Verbeek H, et al. "There's No Place Like Home": A Scoping Review on the Impact of Homelike Residential Care Models on Resident-, Family-, and Staff-Related Outcomes. J Am Med Dir Assoc 2016. 17: 685-93.

27. European Quality Framework for long-term care services. Principles and guidelines for the wellbeing and dignity of older people in need of care and assistance. 2020. hhtp www.wedo-partnership.eu. Accessed May 15th 2020.

28. Beswick AD, Rees K, Dieppe P, Ayis S, Gooberman-Hill R, Horwood J, et al Complex interventions to improve physical function and maintain independent living in elderly people: a systematic review and meta-analysis. Lancet 2008; 37:725-35

29. Grabowski DC, O'Malley AJ. Use Of Telemedicine Can Reduce Hospitalizations Of Nursing Home Residents And Generate Savings For Medicare. Health Aff 2014 33:244-50. 